\title{
Novas perspectivas no tratamento do GIST
}

\author{
New perspectives in the treatment of GIST
}

\author{
Marcus Valadão', Eduardo Linhares², Jorge Mali Jr , Jairo Sousa ${ }^{3}$, Gustavo Stoduto ${ }^{3}$
}

\section{Resumo}

O conhecimento gerado nos últimos anos a respeito dos mecanismos moleculares envolvidos na patogênese do GIST foi responsável pelo avanço no campo do diagnóstico e tratamento desta neoplasia. Só, recentemente, pôdese identificar o GIST como uma entidade clínica distinta e compreender algumas características de sua biologia e história natural. Observou-se, historicamente, que a cirurgia representava a única modalidade terapêutica efetiva. Porém, os resultados com a cirurgia isolada mostraram-se desanimadores, visto que apenas 30 a $40 \%$ dos pacientes estariam curados após longo acompanhamento. A descoberta do imatinib trouxe benefícios para os casos de doença metastática e irressecável, produzindo resultados animadores e trazendo à tona novas perspectivas de sua utilização como tratamento adjuvante/neoadjuvante, visando a otimizar os resultados da cirurgia e aumentar as chances de cura para os portadores dessa neoplasia.

Palavras-Chave: Tumores do estroma gastrointestinal, Cirurgia, Imatinib, Tratamento, Sobrevida, Recidiva.

\section{Abstract}

The insight gained in recent years concerning the molecular pathogenesis of gastrointestinal stromal tumor (GIST) has led to advances in knowledge about its diagnosis and treatment. Only recently has GIST been understood as a distinct clinical entity, with its biological features and natural history recognized. Historically, surgery was the only effective treatment. However, surgical outcome was disappointing, since only $30-40 \%$ of patients were cured after long-term follow-up. The discovery of imatinib benefited patients with metastatic and unresectable disease, providing hopeful results and bringing new perspectives that its combined use with surgery could improve outcome and increase the probability of cure.

Key words: GIST, Surgery, Imatinib, Treatment, Survival, Recurrence.

\footnotetext{
${ }^{1}$ Cirurgião oncológico do INCA. Membro Adjunto do Colégio Brasileiro de Cirurgiōes.

${ }^{2}$ Cirurgião da Seção de Cirurgia Abdômino-Pélvica-INCA. Mestre e Doutor em cirurgia abdominal.

${ }^{3}$ Residente de cirurgia oncológica da Seção de Cirurgia Abdômino-Pélvica-INCA.

Enderę̧o para correspondência: E-mail: marcusvaladao@terra.com.br
} 


\section{INTRODUÇÃO}

Os tumores estromais do trato gastrointestinal (GIST, sigla em inglês para gastrointestinal stromal tumors) representam $80 \%$ dos tumores mesenquimais gastrointestinais, $5 \%$ de todos os sarcomas e $3 \%$ de todas as neoplasias do trato digestivo. ${ }^{1}$ No passado, o GIST era classificado como leiomioma, leiomiossarcoma, leiomioblastoma ou tumor derivado do plexo autonômico e só, recentemente, foi esclarecido que esta neoplasia constitui uma entidade bem definida (origem nas células intersticiais de Cajal e expressão da proteína Kit). ${ }^{2}$

As descobertas geradas, nos últimos cinco anos, a respeito dos mecanismos moleculares envolvidos na patogênese do GIST foram responsáveis pelo avanço do conhecimento no diagnóstico e tratamento desta neoplasia ${ }^{3}$. A incidência do GIST parece ser maior do que a que se pensava, em parte pela maior acurácia na sua identificação (positividade para c-Kit por imunoistoquímica) e pela difusão do conhecimento sobre essa doença no meio médico, permitindo o reconhecimento de maior número de casos conseqüente ao maior índice de suspeição por parte dos profissionais envolvidos.

Kit é um receptor tirosina quinase transmembrana no qual a porção extracelular se liga ao fator de célula tronco Stem-cell factor, também conhecido como fator Steel $l^{4}$ e sua porção intracelular contém o domínio quinase enzimático. A ativação do Kit normalmente ocorre quando dois receptores adjacentes são unidos pela ligação ao dímero do fator de células tronco. Esse processo, conhecido como homodimerização, é acompanhado de mudanças estruturais no receptor, resultando em ativação do domínio quinase do receptor Kit. A quinase ativada fosforila os resíduos de tirosina do homodímero oposto, levando a alterações adicionais à estrutura do receptor Kit, ativando-o. As fosfotirosinas também servem como sítio de ligação para várias proteínas responsáveis pela sinalização celular, muitas das quais são fosforiladas pelo receptor Kit. Esses passos culminam na ativação da complexa cascata de sinalização celular que controla funções celulares cruciais na tumorigênese, incluindo proliferação, adesão, apoptose e diferenciação.

O Kit é expresso nas células tronco hematopoiéticas, nos melanócitos, nas células germinativas, nos mastócitos e nas células intersticiais de Cajal. Notadamente, a expressão da proteína Kit tem papel crucial embriologicamente, influenciando na diferenciação das células progenitoras primitivas mesenquimais em células intersticiais de Cajal (CIC). Além disso, a expressão da proteína Kit é requisito para proliferação das CIC precursoras e para diferenciação e proliferação das CIC, o que é essencial para formação de uma rede de CIC funcionantes..$^{5-7}$ Nos tumores estromais gastrointestinais (GIST), a mutação no gene Kit leva a uma ativação constitutiva na proteína Kit, independente da presença do seu ligante (fator de células-tronco). Conseqüentemente, há um estímulo contínuo para proliferação celular.

A maioria dos GIST apresenta mutação com ganho de função do gen Kit, ${ }^{2}$ sendo mais freqüente a mutação no éxon 11 , que codifica a proteína da região justamembrana intracelular ${ }^{8}$ do receptor tirosina quinase. Outras mutações (éxons 9,13,14 e 17) foram identificadas, ${ }^{2}$ porém numa freqüência menor. Singer et al. ${ }^{9}$ evidenciaram mutação do éxon $11 \mathrm{em} 71 \%$ dos casos, $13 \%$ no éxon $9,4 \%$ no éxon 13 e $4 \%$ no éxon 17 . O tipo de mutação tem sido implicado como fator prognóstico, sendo descrito por Heinrich et al. ${ }^{10}$ como a melhor resposta ao imatinib e maior sobrevida livre de doença nos pacientes portadores de mutação do éxon 11 comparado aos com mutação nos éxons 9, 13 e 17 em estudo fase II com alocação de 127 pacientes. A mutação no éxon 11 representou o fator preditor mais importante de resposta ao imatinib, reduzindo o risco relativo de progressão em $83 \%$ e o risco relativo de morte em $96 \%$.

Esse conhecimento serviu de suporte para publicação de Corless et al., ${ }^{11}$ em que os autores propõem uma nova classificação molecular do GIST, baseando-se no tipo de mutação presente no tumor (Tabela 1), visto que essa classificação teria implicações tanto diagnósticas como terapêuticas.

O diagnóstico de GIST é feito quando existem características morfológicas celulares típicas associadas à imunoistoquímica positiva para Kit. Porém, alguns tumores (estimado entre 2 e $10 \%)^{11}$ apresentam características clínicas e patológicas compatíveis com GIST, mas não expressam a proteína Kit. Heinrich et al. ${ }^{12}$ demonstraram que este subgrupo (GIST c-Kit negativo) apresenta mutação com ganho de função em um receptor tirosina quinase com atividades semelhantes ao Kit, denominado Receptor alfa do fator de crescimento derivado das plaquetas (PDGFR $\alpha$ ), constituindo-se numa via alternativa na patogênese desta neoplasia. Sihto et al..$^{13}$ evidenciaram que, apesar da expressão da proteína Kit (identificação por imunoistoquímica) em vários outros tipos histológicos, apenas os GIST apresentavam mutações no gen Kit e PDGFR $\alpha$, utilizando-se técnicas de seqüenciamento de DNA e desnaturação pela cromatografia líquida de alta performance.

O estadiamento ao diagnóstico é um dos fatores prognósticos mais importantes, visto que a presença de doença metastática é marcador de sobrevida reduzida. ${ }^{14}$ $\mathrm{Na}$ ausência de metástase, o prognóstico tende a se correlacionar fundamentalmente com duas características: tamanho do tumor e atividade mitótica, baseados em dados consistentes da literatura. ${ }^{1,15-18}$ 
Tabela 1. Classificação molecular do GIST (Corless et al. ${ }^{11}$ )

\begin{tabular}{|c|c|}
\hline Tipo de GIST & Comentários \\
\hline $\begin{array}{l}\text { GIST ESPORÁDICO } \\
\text { Mutação do gen Kit } \\
\text { Éxon } 11 \\
\text { Éxon } 9 \\
\text { Éxon } 13 \\
\text { Éxon } 17\end{array}$ & $\begin{array}{l}\text { Melhor resposta ao imatinib. } \\
\text { Resposta intermediária ao imatinib. } \\
\text { Sensível in vitro ao imatinib; resposta clínica observada. }\end{array}$ \\
\hline $\begin{array}{l}\text { Mutação do gen PDGFR } \alpha \\
\text { Éxon } 12\end{array}$ & Sensível in vitro ao imatinib; resposta clínica observada. \\
\hline Éxon 18 & $\begin{array}{l}\text { D842V tem resposta ruim ao imatinib; outras mutações são } \\
\text { sensíveis. }\end{array}$ \\
\hline Tipo selvagem (sem mutação) & Resposta ruim ao imatinib. \\
\hline $\begin{array}{l}\text { GIST FAMILIAR } \\
\text { Kit éxon } 11 \text { ( V559A, delV559, W557R) }\end{array}$ & Pigmentação de pele, urticária pigmentosa, mastocitose. \\
\hline Kit éxon $13(\mathrm{~K} 64 \mathrm{E})$ & Sem pigmentação de pele, nem mastocitose. \\
\hline $\begin{array}{l}\text { Kit éxon } 17(\mathrm{D} 820 \mathrm{Y}) \\
\text { GIST com paraganglioma }\end{array}$ & $\begin{array}{l}\text { Sem pigmentação de pele, nem mastocitose; } \\
\text { anormalidade na peristalse esofágica. } \\
\text { Autossômico dominante; sintomas endócrinos são comuns. }\end{array}$ \\
\hline $\begin{array}{l}\text { GIST PEDIÁTRICO } \\
\text { Esporádico } \\
\text { Tríade de Carney }\end{array}$ & $\begin{array}{l}\text { Mutação no Kit (muito menos comum do que em adultos). } \\
\text { GIST gástrico com condroma pulmonar com ou sem } \\
\text { paraganglioma; mutações Kit não identificadas. }\end{array}$ \\
\hline $\begin{array}{l}\text { GIST relacionado à } \\
\text { neurofibromatose tipo I }\end{array}$ & Mutações Kit não identificadas. \\
\hline
\end{tabular}

Apesar da existência de inúmeros fatores prognósticos descritos na literatura (rotura tumoral durante a cirurgia, localização anatômica, presença de necrose intratumoral, tipo de mutação do gen Kit, marcadores de proliferação celular, dentre outros), a predição do comportamento biológico do GIST é incerta. Conseqüentemente, tem-se evitado o termo "benigno" e classificado o GIST de acordo com o potencial de malignidade com base nos dois fatores prognósticos mais relevantes (Tabela 2).

\section{TRATAMENTO DE DOENÇA RESSECÁveL}

A ressecção cirúrgica completa é o tratamento padrão para o GIST, pois é a única modalidade capaz de proporcionar cura. A ressecção R0 (ausência de doença residual) representa uma das influências mais
Tabela 2. Estimativa do potencial de malignidade (Fletcher et al. ${ }^{19}$ )

\begin{tabular}{lcc}
$\begin{array}{c}\text { Risco de } \\
\text { malignidade }\end{array}$ & Tamanho $(\mathrm{cm})$ & $\begin{array}{c}\text { Índice Mitótico } \\
\text { (50cga) }\end{array}$ \\
\hline Muito Baixo & $<2$ & $<5$ \\
Baixo & $2-5$ & $<5$ \\
Intermediário & $<5$ & $6-10$ \\
& $5-10$ & $<5$ \\
Alto & $>5$ & $>5$ \\
& $>10$ & Qualquer Índice \\
& $\begin{array}{l}\text { Qualquer } \\
\text { Tamanho }\end{array}$
\end{tabular}


importantes para o resultado do tratamento (intervalo livre de doença e sobrevida), sendo alcançada em torno de 40 a $60 \%$ de todos os casos de GIST e em mais de $70 \%$ nos casos de doença não metastática. ${ }^{1,20} \mathrm{O}$ objetivo primordial da cirurgia é a ressecção completa do tumor, geralmente sendo possível através da ressecção segmentar do sítio de origem da lesão (principalmente no GIST de localização gástrica), obtendo-se margens negativas de $1 \mathrm{a} 2 \mathrm{~cm}$ avaliadas por exame de congelação intraoperatória. As lesões com suspeita de invasão de órgãos adjacentes devem ser tratadas por cirurgia radical através da ressecção em monobloco do órgão acometido. É necessária uma técnica cirúrgica meticulosa visando a prevenir a rotura tumoral durante o ato cirúrgico, pois a cápsula do tumor se rompe com facilidade, podendo resultar em disseminação neoplásica e pior prognóstico. ${ }^{21} \mathrm{~A}$ metástase nodal é um evento infreqüente, ${ }^{1}$ não havendo subsídio na literatura que corrobore a realização de linfadenectomia de rotina.

O acompanhamento em longo prazo de portadores de GIST de alto risco submetidos à ressecção cirúrgica indica que a cirurgia isolada geralmente não é capaz de proporcionar cura, pois 85 a $90 \%$ desses casos irão desenvolver algum efeito adverso (recidiva, metástase ou morte). ${ }^{19}$ Em geral, mais de $50 \%$ dos pacientes submetidos à cirurgia potencialmente curativa irão desenvolver recidiva ou doença metastática ${ }^{20,22}$ e irão cursar com sobrevida reduzida.

\section{TRATAMENTO DA RECIDIVA}

A presença de recidiva é freqüente apesar da ressecção cirúrgica completa do tumor primário. $\mathrm{Na}$ experiência do MD Anderson Cancer Center, somente $10 \%$ dos pacientes estavam livres de doença após 10 anos de acompanhamento. ${ }^{21} \mathrm{O}$ padrão inicial de recidiva envolve, predominantemente, o peritônio e o fígado. $\mathrm{Na}$ casuística do Memorial Sloan-Kattering Cancer Center, $40 \%$ dos pacientes submetidos à cirurgia potencialmente curativa (R0) desenvolveram recidiva, com acometimento do peritôneo em $50 \%$ dos casos e do fígado em 75\%, tendo sobrevida média de 15 meses após resgate cirúrgico. ${ }^{23}$

O resgate cirúrgico deve ser empregado sempre que seja constatada doença ressecável, porém todos esses pacientes irão desenvolver recidivas subseqüentes apesar da ressecção macroscópica completa dessas lesões. A recidiva peritoneal, geralmente, permite a ressecção completa do tumor, porém a recorrência hepática usualmente se apresenta como doença difusa intrahepática, impedindo a abordagem cirúrgica.

\section{TRATAMENTO DA DOENÇA METASTÁTICA}

A abordagem cirúrgica da doença metastática está restrita a um número limitado de casos, visto que a grande maioria dos pacientes com doença metastática apresenta disseminação extensa, impossibilitando a ressecção tumoral. Diante deste fato, alguns tipos de tratamento foram utilizados para controlar doença metastática irressecável como quimioterapia sistêmica ou intraperitoneal e a quimioembolização da artéria hepática, porém sem evidência de benefício. ${ }^{24}$

A descoberta do STI571 (mesilato de imatinib [Glivec ${ }^{\circledR}$; Novartis, Basel, Switzerland) revolucionou o tratamento do câncer por ser a primeira terapia a agir especificamente na alteração molecular responsável pela etiologia do câncer. O conhecimento de mutações (com ganho de função do gene Kit $)^{2}$ na gênese e progressão do GIST proporcionou o desenvolvimento de uma droga com alvo molecular definido que interfere na atividade tirosina quinase dos receptores Kit. Inicialmente utilizado no tratamento da leucemia mielóide crônica com grande sucesso, o STI571 também começou a ser usado no tratamento dos GIST.

O primeiro relato de tratamento do GIST com STI571 foi em fevereiro de 2000, na Finlândia. ${ }^{25} \mathrm{~A}$ paciente apresentava doença disseminada na cavidade abdominal, tendo insucesso com várias outras modalidades terapêuticas. $\mathrm{O}$ tumor expressava a proteína Kit (imunoistoquímica positiva para CD117) e continha mutação no éxon 11 do gene Kit. Em algumas semanas de tratamento, houve resposta dramática mensurada por tomografia, PET scan e biópsias seriadas do tumor. Histologicamente, o tumor foi substituído por degeneração mixóide e fibrose.

Os resultados encorajadores com o primeiro caso levaram à implementação de estudos fases I e II confirmando sua atividade. ${ }^{26,27} \mathrm{O}$ estudo fase I realizado pela EORTC ${ }^{26}$ contendo 36 pacientes concluiu que a maior dose tolerada de mesilato de imatinib era de $400 \mathrm{mg}$ duas vezes ao dia, tendo atividade significante nos doentes com GIST avançado e que essa terapia não beneficiou pacientes com outros tipos de sarcomas que expressavam Kit. Baseado neste estudo fase I, foi realizado um estudo multicêntrico fase II sobre a utilização do Glivec $^{\circledR}$ para o tratamento do GIST irressecável ou metastático. ${ }^{27}$ Este estudo randomizou 147 doentes para receber 400 ou $600 \mathrm{mg} /$ dia da droga. Ocorreu toxicidade grau 3 ou 4 (hemorragia, dor abdominal, distúrbio eletrolítico) em $21,1 \%$ dos casos. O acompanhamento mediano foi de 288 dias, a taxa de resposta parcial (diminuição de pelo menos $50 \%$ do tamanho da lesão) ocorreu em $53,7 \%$ e a estabilização da doença ocorreu em $27,9 \%$. Treze por cento dos casos apresentaram progressão de doença. O tempo médio de resposta objetiva foi de 13 semanas e não houve 
diferença significante em toxicidade e resposta entre o grupo que utilizou $400 \mathrm{mg}$ e o que utilizou $600 \mathrm{mg}$.

Devido à eficácia deste tratamento, constatado em estudos fases I e II, foram realizados estudos fase III comparando o uso do Glivec ${ }^{\circledR}$ em doses de 400 e $800 \mathrm{mg} /$ dia em pacientes com GIST metastático. O estudo europeu multicêntrico ${ }^{28}$ randomizou 946 pacientes (portadores de GIST metastático) com a finalidade de comparar a sobrevida livre de progressão entre o grupo que utilizou $400 \mathrm{mg} / \mathrm{dia}$ e $800 \mathrm{mg} / \mathrm{dia}$ de imatinib. Os resultados desse trabalho evidenciaram que a resposta clínica foi semelhante entre os grupos e que a dose de $800 \mathrm{mg} /$ dia promoveu significativo aumento na sobrevida livre de progressão.

\section{NOVAS PERSPECTIVAS}

É fato que o tratamento cirúrgico isolado do GIST não metastático é insatisfatório em uma grande proporção de casos, visto que $50 \%$ dos pacientes submetidos à ressecção potencialmente curativa irão desenvolver recidiva ou metástase à distância, ${ }^{20,22}$ especialmente no subgrupo de alto risco em que essas taxas se elevam para 85 a $90 \% .^{20}$

Se por um lado está claro que o imatinib traz benefícios aos pacientes com doença irressecável ou metastática, por outro, vem à tona o questionamento se o imatinib seria benéfico no subgrupo de pacientes operados que tivessem prognóstico ruim. Essa hipótese despertou o início da utilização do imatinib como terapia adjuvante e neoadjuvante, como instrumento capaz de otimizar os resultados obtidos com a cirurgia.

$\mathrm{O}$ racional é que a terapia adjuvante/neoadjuvante com imatinib seria capaz de reduzir as chances de recidiva local e de metástase à distância, prolongar o intervalo livre de doença e a sobrevida global, aumentar o número de casos irressecáveis elegíveis para ressecção (redução tumoral farmacológica) e otimizar a resposta ao imatinib após citorredução cirúrgica. ${ }^{3}$

Algumas linhas de pesquisa estão em andamento com o intuito de esclarecer essas questôes. Dentre elas, destaca-se o estudo fase II do Grupo de Oncologia do Colégio Americano de Cirugiōes (ACOSOG-Z9000), ${ }^{29}$ que tem como finalidade avaliar sobrevida, taxa de recidiva e toxicidade após tratamento adjuvante com imatinib por um ano em pacientes de alto risco (definidos como tamanho tumoral maior do que $10 \mathrm{~cm}$, rotura tumoral, hemorragia intra-peritoneal, tumores intra-peritoneais multifocais) submetidos à ressecção completa. $\mathrm{O}$ estudo ainda não foi concluído, mas os resultados preliminares apresentados no encontro anual da Sociedade Americana de Oncologia Clínica (ASCO), em abril de 2005, revelou ${ }^{30}$ toxicidade grau 3 em 17\% dos pacientes e necessidade de redução da dose devido à toxicidade em $22 \%$ dos casos. Oitenta e dois por cento dos pacientes completaram um ano de tratamento com imatinib, demonstrando boa tolerância à droga. Os dados de sobrevida ainda aguardam a conclusão do estudo.

O estudo fase III, multicêntrico, patrocinado também pelo Colégio Americano de Cirurgiōes (ACOSOGZ9001), ${ }^{29}$ objetiva responder, por meio da randomização de 380 casos, se existe benefício, em longo prazo, com a utilização do imatinib adjuvante em pacientes portadores de GIST de risco intermediário operados. O grupo de sarcoma ósseo e de partes moles da EORTC ${ }^{29}$ também está avaliando os resultados da terapia adjuvante com imatinib, sendo projetada a alocação de 400 casos para o ensaio clínico. O ensaio clínico do grupo de sarcoma escandinavo (SSGXVII) ${ }^{29}$ espera concluir seu estudo multicêntrico, randomizado, com 80 casos de GIST distribuídos em dois braços (400 mg de imatinib adjuvante por 12 ou 36 meses).

O tratamento neoadjuvante com imatinib também está sendo testado, tendo como exemplo o estudo RTOGS0132 $2^{29}$, visando a avaliar o impacto dessa abordagem na sobrevida livre de progressão e na taxa de resposta objetiva.

A conclusão destes trabalhos trará, em breve, respostas valiosas a respeito da promissora associação cirurgia $\mathrm{x}$ imatinib no tratamento dessa intrigante neoplasia. Além disso, o desenvolvimento de novas drogas, capazes de agirem nos diferentes mecanismos moleculares implicados na patogênese do GIST (AMG 706, SU11248) ${ }^{31,32,33}$, associado ao conhecimento da classificação molecular baseada na identificação dos tipos de mutação11, permitirá no futuro o tratamento específico e individualizado para cada situação molecular.

\section{REFERÊNCIAS}

1. De Matteo RP, Lewis JJ, Leung D, Muddan SS, Woodruff JM, Brennan MF. Two hundred gastrointestinal stromal tumors: recurrence patterns and prognostic factors for survival. Ann Surg. 2000;231:51-8.

2. Hirota S, Isozaki K, Moriyama Y, Hashimoto K, Nishida T, Ishiguro $S$, et al. Gain-of-function mutations of c-Kit in human gastrointestinal stromal tumors. Science. 1998;279:577-80.

3. D'Amato G, Steinert DM, McAuliffe JC, Trent JC. Update on the biology and therapy of gastrointestinal stromal tumors. Cancer Control. 2005;2:44-56.

4. Majumder S, Brown K, Qiu FH. C-Kit protein, a transmembrane kinase: Identification in tissues and characterization. Mol Cell Biol. 1988;8:4896-4903.

5. Huizinga JD, Thuneberg L, Kluppel M, Malysz J, Mikkelsen HB, Bernstein A. W/Kit gene required for 
intestinal cells of Cajal and for intestinal pacemaker activity. Nature. 1995;373:347-9.

6. Isozaki K, Hirota S, Nakama A. Disturbed intestinal movement, bile reflux to the stomach, and deficiency of cKit expressing cells in Ws/Ws mutant rats. Gastroenterology. 1995;109:456-64.

7. Torihashi S, Nishi K, Tokutomi Y, Nishi T, Ward S, Sanders KM. Blockade of Kit signaling induces transdifferentiation of intersticial cells of Cajal to a smooth muscle phenotype. Gastroenterology. 1999;117:140-8.

8. Heinrich MC, Corless CL, Demetri GD, Blanke CD, von Mehren M, Joensu H, et al. Inhibition of Kit tyrosine kinase activity: A novel molecular approach to the treatment of Kitpositive malignances. J Clin Oncol. 2002;20:1692-1703.

9. Singer S, Rubin BP, Lux ML, Chen CJ, Demetri GD, Fletcher CDM, et al. Prognostic value of Kit mutation type, mitotic activity, and histologic subtype in gastrointestinal stromal tumors. J Clin Oncol. 2002;20:3898-3905.

10. Heinrich MC, Corless CL, Demetri GD, Blanke CD, von Mehren M, Joensuu H, et al. Kinase mutations and imatinib response in patients with metastatic gastrointestinal stromal tumors. J Clin Oncol. 2003;21:4342-9.

11. Corless CL, Fletcher J, Heinrich MC. Biology of gastrointestinal stromal tumors. J Clin Oncol. 2004;22:3813-25.

12. Heinrich MC, Corless CL, Duesing A, McGreevey L, Chen CJ, Joseph $\mathrm{N}$, et al. PDGFRA activating mutations in gastrointestinal stromal tumors. Science. 2003;299:708-10.

13. Sihto H, Sarmolo-Rikala M, Tynninen O, Tanner M, Andersson L, Franssila K, et al. Kit and Platelet-Derived Growth Factor Receptor Alpha Tyrosine Kinase gene mutations and Kit amplifications in human solid tumors. J Clin Oncol. 2005;23:49-57.

14. Graadt van Roggen JF, van Velthuysen MLF, Hogendoorn PCW. The histopathological differential diagnosis of gastrointestinal stromal tumors. J Clin Pathol. 2001;54:96-102.

15. Valadão M, Linhares E, Castro L, Pinto CE, Lugão R, Quadros C, et al. GIST gástrico-Experiência do INCA. Rev Bras Cancerol. 2004;50(2):121-6.

16. Fujimoto Y, Nakanishi Y, Yoshimura K, Shimoda T. Clinicopathologic study of primary malignant gastrointestinal stromal tumor of the stomach, with special reference to prognostic factors: analysis of results in 140 surgically resected patients. Gastric Cancer. 2003;6(1):39-48.

17. Mochizuki Y, Kodera Y, Ito S, Yamamura Y, Kanemitsu Y, Shimizu Y, et al. Treatment and risk factors for recurrence after curative resection of gastrointestinal stromal tumors of the stomach. World J Surg. 2004;28(9):870-5.

18. Wong NACS, Young R, Malconson RDG, Nayar AG, Jamieson LA, Save VE, et al. Prognostic indicators for gastrointestinal stromal tumors: a clinicopathological and immunohistochemical study of 108 resected cases of the stomach. Histopathol. 2003;43:118-26.

19. Fletcher CD, Bermen JJ, Corless C, Gorstein F, Lasota J, Longley BJ, et al. Diagnosis of gastrointestinal stromal tumors: a consensus approach. Hum Pathol. 2002;33:459-65.

20. Roberts PJ, Eisenberg B. Clinical presentation of gastrointestinal stromal tumors and treatment of operable disease. Eur J Cancer. 2002; 38 Suppl 5:S37-8

21. Ng EH, Pollock RE, Munsell MF, Atkison EN, Romsdahl MM. Prognostic factors influencing survival in gastrointestinal leiomyosarcomas: implications for surgical management and staging. Ann Surg. 1992;215:68-77.

22. Lehnert T. Gastrointestinal sarcoma (GIST): a review of surgical management. Ann Chir Gynaecol. 1998;87:297-305.

23. Mudan SS, Conlon KC, Woodruff J, Lewis J, Brennan MF. Salvage surgery in recurrent gastrointestinal sarcoma: Prognostic factors to guide patient selection. Cancer. 2000;88:66-74.

24. Pidhorecky I, Cherney RT, Kraybill WG, Gibbs JF. Gastrointestinal stromal tumors: current diagnosis, biologic behavior and management. Ann Surg Oncol. 2000;7:705-12.

25. Joensuu H, Roberts PJ, Sarlomo-Rikala M, Anderson LC, Tervahartiala P, Tuveson D, et al. Effect of tyrosine kinase inhibitor STI571 in a patient with a metastatic gastrointestinal stromal tumor. $\mathrm{N}$ Engl J Med. 2001;344:1052-6.

26. van Oosterom AT, Judson I, Verweij J, Stroobants S, di Paola ED, Dimitrijevic S, et al. Safety and efficacy of imatinib (STI571) in metastatic gastrointestinal stromal tumors: a phase I study. Lancet. 2001;358:1421-3.

27. Demetri GD, von Mehren M, Blanke CD, Van den Abbeele AD, Eisenberg B, Roberts PJ, et al. Efficacy and safety of imatinib mesylate in advanced gastrointestinal stromal tumors. N Engl J Med. 2002;347:472-80.

28. Verweij J, Casali PG, Zalcberg J, LeCesne A, Reichardt P, Blay JY, et al. Progression-free survival in gastrointestinal stromal tumours with high-dose imatinib: randomised trial. Lancet. 2004;364:1127-34.

29. Eisenberg B, Judson I. Surgery and imatinib in the management of GIST: Emerging approaches to adjuvant and neoadjuvant therapy. Ann Surg Oncol. 2004;11:465-75.

30. DeMatteo RP, Antonescu CR, Chadaram V, You YN, McCall L, Maki A, et al. Adjuvant imatinib mesylate in patients with primary high risk gastrointestinal stromal tumor (GIST) following complete resection: safety results from the U.S. Intergroup Phase II trial ACOSOG Z9000. Proc Am Soc Clin Oncol. 2005; abstr 9009.

31. Herbst RS. A phase I, first in human, open-label, dose finding study evaluating the safety and pharmacokinetics of AMG 706 in subjecys with advanced solid tumors [citado em 2005 Out 23]. Disponível em: http://utmext01a.mdacc.tmc.edu/dept/prot/clinicaltrialswp.nsf/ index/2003-0460. Acesso em outubro de 2005. 
32. Maki RG, Fletcher JA, Heinrich MC, Morgan JA, George S, Desai J, et al.Results from a continuation trial of SU11248 in patients with imatinib resistant gastrointestinal stromal tumors (GIST). Proc Am Soc Clin Oncol 2005; abstr 9011.
33. Demetri GD, Heinrich MC, George S. Biological activity of the multi-targeted tyrosine kinase inhibitor SU11248 in patients with malignant gastrointestinal stromal tumors (GIST). Cancer Res. 2003;44:1114. 